\title{
Trajectories of depressive symptoms in response to prolonged stress in medical interns
}

Guille C, Clark S, Amstadter AB, Sen S. Trajectories of depressive symptoms in response to prolonged stress in medical interns.

Objective: The high degree of heterogeneity in the development of depression under stress is unaccounted for in traditional statistical modeling. We employ growth mixture modeling to identify classes of individuals at highest risk of depression under stress.

Method: Medical internship was used as a prospective stress model. Interns from US residency programs completed demographic, psychological, and depressive symptom assessments 2 months prior to internship and at 3-month intervals throughout internship year. Results: A total of 2278 (59\%) of interns chose to take part in the study. Three classes of depressive symptoms were identified: i) Stressresilient class: $62 \%$ of participants report low depressive symptoms before and throughout internship year; ii) Stress-neutral class: $22 \%$ of participants report mild depressive symptoms before and throughout internship year; and iii) Stress-sensitive class: $16 \%$ of participants report low depressive symptoms before internship stress, and high levels of depressive symptoms throughout internship year. Individuals in the Stress-sensitive class were more likely to be female, in a surgical specialty, and have a history of depression, difficulty early family environment and high-neuroticism scores compared with individuals in the Stress-resilient class.

Conclusion: Trajectory-based analysis allows for the identification of a high-risk group, within a heterogeneous population, that accounts for the link between stress and depression.

\section{Guille ${ }^{1}$, S. Clark ${ }^{2}$, A. B. Amstadter ${ }^{3}$, S. Sen ${ }^{4}$}

${ }^{1}$ Department of Psychiatry and Behavioral Sciences, Medical University of South Carolina, Charleston, SC, ${ }^{2}$ Center for Biomarker Research and Personalized Medicine, Virginia Commonwealth University School of Pharmacy, ${ }^{3}$ Department of Psychiatry, Virginia Institute of Psychiatric and Behavioral Genetics, Virginia Commonwealth University, Richmond, VA and ${ }^{4}$ Department of Psychiatry, University of Michigan, Ann Arbor, MI, USA

Key words: affective disorders; stress; biostatistics Constance Guille, Department of Psychiatry and Behavioral Sciences, Medical University of South Carolina, 67 President St. MSC861, 5 South, Charleston, SC 29425, USA.

E-mail: guille@musc.edu

Accepted for publication March 8, 2013

\section{Significant outcomes}

- Growth mixture modeling identifies and characterizes distinct classes of depressive symptoms under conditions of stress and a number of important prestress predictors of class membership.

- Our findings suggest that the increase in depressive symptoms in response to stress is a precipitant to depressive symptoms in only a small subsample of individuals, rather than a common increase across the full population as suggested by previous studies.

- The identification of a high-risk group has important clinical and health economic implications as it allows the focus of limited prevention and intervention resources on the small subset of individuals most vulnerable to responding poorly to stress.

\section{Limitations}

- Only $59 \%$ of potential subjects agreed to take part in the study.

- Depressive symptoms were assessed through a self-report inventory rather than a diagnostic interview.

- Our study was restricted to interns completing their first year of postgraduate medical training. Thus, it is unknown if similar classes of depressive symptom trajectories hold true for other populations experiencing a stressor. 


\section{Guille et al.}

\section{Introduction}

Major depression affects approximately $16 \%$ of Americans at some point in life (1). According to the World Health Organization, depression is far and away the leading cause of disease-associated disability among individuals 15-44 years old. Life stress is one of the strongest, most consistent risk factors for major depression $(2,3)$ with approximately $80 \%$ of depressive episodes preceded by a major stressor (4). However, the majority of individuals encountering stressful life events do not become depressed, indicating that there is variation in how individuals respond to stress (5).

The high degree of heterogeneity in the development of depression in response to stress constitutes an important public health problem, with significant implications for how to effectively focus resources on those individuals at highest risk of depression under stress. A large body of work has examined demographic and psychological factors that correlate with the development of depression under stress and has advanced our understanding of this condition (6-8). However, drawing conclusions about the relationship between these factors and symptoms of depression is often not possible due to limitations imposed by the methodology employed (e.g., retrospective or cross-sectional study designs which cannot distinguish the cause and effect relationship between potential risk factors and depression under stress) (9). There are a limited number of longitudinal studies assessing the relationship between stress and depression, and most of these have not gathered baseline psychological data from before the onset of stress (10, 11). Further, these studies have employed a mixedeffects growth model, which assumes the same mean trajectory of depressive symptoms over time for all participants. This approach tends to obscure interindividual heterogeneity and cannot identify distinct trajectories of depressive symptoms under stress (9). In contrast, trajectory-based models (also known as growth mixture modeling or latent class growth models) allow for the identification of distinct classes of developmental trajectories of depressive symptoms and the identification of factors that predict class membership.

In this study, we aimed to overcome prior study limitations by utilizing a trajectory-based model to examine depressive symptom trajectory classes and predictors of class membership before and during a naturalistic prolonged stressor, medical internship. Medical internship is a rare situation where a dramatic increase in stress and depressive symptoms can be predicted $(12,13)$.
Aims of the study

This study sought to i) determine individual trajectories of depressive symptoms over the course of medical internship via employment of growth mixture modeling; and ii) identify personal factors that predict trajectory class membership.

\section{Material and methods}

Participants

A total of 4175 interns entering traditional internal medicine, general surgery, pediatrics, and psychiatry residency programs during the 2007, 2008, 2009, and 2010 academic years were sent an email 2 months prior to commencing internship and invited to participate in the study. For 314 subjects, our email invitations were returned as undeliverable, and we were unable to obtain a valid email address. Of those with a valid email address, $59 \%(2278 / 3861)$ of the remaining invited subjects agreed to participate in the study (Table 1). The Institutional Review Board at participating hospitals approved the study. Subjects were given $\$ 30$ (2007 cohort) or $\$ 40$ (2008, 2009, and 2010 cohorts) gift certificates for their participation.

\section{Data collection}

All surveys were conducted through a secure online website designed to maintain confidentiality, with subjects identified only by numbers. No links between the identification number and the subjects' identities were maintained.

Depressive symptoms were measured using the depression module of the Patient Health Questionnaire (PHQ-9). The PHQ-9 is a self-report component of the PRIME-MD inventory designed to

Table 1. Descriptive data for covariates

\begin{tabular}{lcr}
\hline & \multicolumn{2}{c}{ Baseline } \\
\cline { 2 - 3 } & $n$ & $\%$ \\
\hline Age [Mean, (SD)] & 27.74 & 3.1 \\
Female & 1049 & 45.4 \\
European American & 1280 & 55.4 \\
African American & 83 & 3.6 \\
Latino & 85 & 3.7 \\
Asian & 556 & 24.1 \\
Multi-racial or other & 220 & 9.5 \\
Internal medicine & 948 & 41.0 \\
Surgery & 231 & 10.0 \\
Pediatrics & 259 & 11.2 \\
Psychiatry & 163 & 7.1 \\
Positive history of depression & 995 & 43.1 \\
Neuroticism & 22.15 & 8.80 \\
Early family environment & 15.71 & 9.34 \\
\hline
\end{tabular}


screen for depressive symptoms (14). For each of the nine depressive symptoms, interns indicated whether, during the previous 2 weeks, the symptom had bothered them 'not at all,' 'several days,' 'more than half the days,' or 'nearly every day.' Each item yields a score of $0-3$, so that the PHQ-9 total score ranges from 0 to 27. PHQ scores of five or greater, 10 or greater, 15 or greater, and 20 or greater correspond to mild, moderate, moderately severe, and severe depression, respectively (12). Scores below five are low and considered not clinically relevant. A score of 10 or greater on the PHQ-9 has a sensitivity of $93 \%$ and a specificity of $88 \%$ for the diagnosis of major depressive disorder (15). Diagnostic validity of the PHQ-9 is comparable with clinician-administered assessments (14).

\section{Assessments}

Subjects completed a baseline survey 2 months prior to commencing internship that assessed general demographic factors (age, sex, ethnicity, marital status), medical education factors (medical specialty), personal factors (baseline PHQ-9 depressive symptoms, self-reported history of depression), and psychological measures (Neuroticism (NEO-Five Factor Inventory) (16), early family environment (Risky Families Questionnaire) (17). and Cognitive Styles (modified SociotropyAutonomy Scale) (18). The Neuroticism subscale of the NEO-Five Factor Inventory evaluates characteristics such as anxiety, moodiness, worry, envy, and jealousy. Higher scores on the Neuroticism subscale indicate an increase likelihood of experiencing negative emotions, especially in response to environmental stress. The Risky Families Questionnaire evaluates early family environment. Higher scores indicate a more chaotic, harsh, and abusive early family environment where lower scores indicate a more organized, supportive, and nurturing early family environment. The Sociotropy-Autonomy Scale assesses two cognitive personality constructs. Individuals with high-sociotropy scores require external validation to experience satisfaction for their achievements, whereas those with high autonomy scores derive greater satisfaction from activities and goals that are achieved independent of others approval. Participants were contacted via email at months $3,6,9$, and 12 of their internship year and asked to complete the PHQ-9.

\section{Statistical analyses}

Mplus Version 6.1 was used to determine distinct classes of depression trajectories via growth mixture modeling with latent variables (19). Growth mixture modeling combines a person-oriented approach with traditional variable-oriented growth curve modeling, permitting continuous latent growth factors (i.e., intercept and slope) to be related to time (19). While conventional growth modeling assumes a single population and estimates a mean growth curve, growth mixture modeling uses categorical latent variables to capture different classes of trajectories within a population. Growth mixture models are estimated with maximum likelihood with robust standard errors, which permits analysis of data missing at random. Evidence for different trajectory classes exists when models with two or more latent classes provide a better fit to the data than models with one class. As the standard chi-square difference test (likelihood ratio test) cannot be used with mixture models (20), information criteria, which compute an index that is a function of the log-likelihood, are commonly used. In this study, we use the Akaike Information Criterion (AIC) (21) and the Bayesian Information Criterion (BIC) (22). The number of latent trajectories was chosen based on the lowest AIC and BIC along with size and substantive interpretation of the classes. Because, as expected, the PHQ score means across did not reflect a linear growth trend, a quadratic model was used. An initial quadratic growth curve analysis on the entire sample of interns (one-class model) provided a point of comparison for increases in numbers of classes. Next, two- to five-class models were assessed. Once the best-fitting number of classes was determined, we tested various submodels that relax the restriction that the growth factor (i.e., intercept, slope, and quadratic term) variances are equivalent across classes. Baseline covariates were added to the model to aid in prediction of individual's trajectory class membership. For the time invariant covariates, a series of multinomial logistic regressions were conducted to determine whether baseline characteristics influence the probability of class membership.

\section{Results}

Consistent with previous studies, there was a dramatic increase in PHQ depression symptom [Mean (SD)] score from baseline [2.45 (3.05)] to 3 months [6.06 (4.87)], 6 months [5.99 (4.75)], 9 months [6.29 (4.98)], and 12 months [5.81 (4.98)] of internship (13). To assess for the presence of distinct trajectory classes, one-through five-class model were tested (Table 2). The four- and five-class solutions did not have a replicated log-likelihood indicating that these solutions were unstable, thus these 


\section{Guille et al.}

Table 2. Model fit and comparison table for quadratic growth mixture models

\begin{tabular}{lcccc}
\hline Number of classes & Log-likelihood & Number of free parameters & AIC & BIC \\
\hline 1 & -21832 & 11 & 43687 & 43750 \\
2 & -21467 & 15 & 42964 & 43050 \\
3 & -21261 & 19 & 42560 & 42669 \\
3A & -21261 & 18 & 42558 & 42661 \\
3B & -20995 & 20 & 42030 & 42144 \\
3C & -20956 & 20 & 41952 & 42066 \\
$4^{*}$ & -21805 & 23 & 42216 & 42347 \\
$5^{*}$ & -21013 & 27 & 42081 & 42235 \\
Models with covariates & & & & \\
3C Time invariant & -18658 & 50 & 37417 & 37694 \\
\hline
\end{tabular}

$N=2240$.

AIC, Akaike Information Criterion; BIC, Bayesian Information Criterion; 3A: Threeclass model with no quadratic variance in all three classes; 3B: Three-class model with no quadratic variance in all three classes, intercept and slope variance freely estimated in Class 2; 3C: Three-class model with no quadratic variance in all three classes, intercept and slope variance freely estimated in Class 2, and no slope factor variance in Class 1.

*Log-likelihood not replicated.

models were rejected. Among the remaining three models, it was determined that three-class solution was the best fitting as indicated by the lowest AIC and $\mathrm{BIC}$ values. We then tested variations of the three-class model that relax the restrictions on the intercept, slope, and quadratic variances equivalency across classes (Models 3A, 3B, and 3C in Table 2), and it was determined that the three-class solution with no quadratic growth factor variance in any class, with the intercept and slope variance freely estimated in Class 2, with no slope factor variance in Class 1, was the best-fitting model as indicated by the AIC and BIC values.

Figure 1 shows the average trajectory in each latent class. Class 1 (Stress-resilient; $62 \%$ of participants) subjects report low depressive symptoms before internship stress and continue with relatively low depressive symptoms through the intern year. Class 2 (Stress-neutral; $22 \%$ of participants) report a mild level of depressive symptoms before internship and continue to report mild symptoms throughout internship. Class 3 (Stress-sensitive; $16 \%$ of participants) subjects report low depressive symptoms before internship, but report a dramatic increase in depressive symptoms with the onset of internship stress.

\section{Predictors of depression trajectory}

To identify variables that predict membership in the different classes, covariates were introduced into the three-class model (Table 3). The introduction of covariates improves model fit as indicated by lower values of the AIC and BIC when compared to the three-class model without covariates (Table 2). A personal history of depression, high

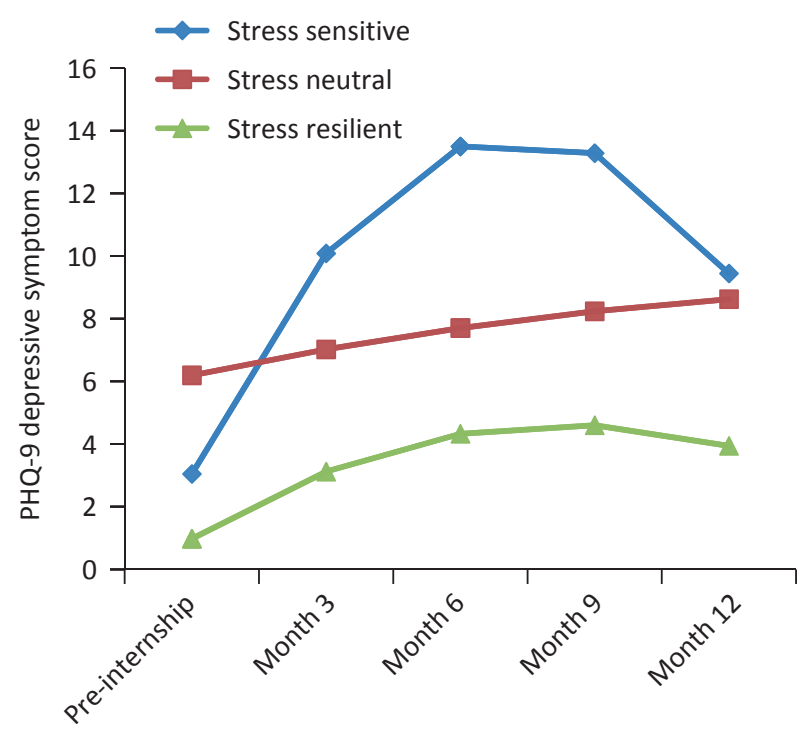

Fig. 1. Mean trajectory of depressive symptoms for three latent classes.

Table 3. Multinomial logistic regression time-invariant covariate effect estimates and standard errors

\begin{tabular}{|c|c|c|c|c|c|c|}
\hline & \multicolumn{2}{|c|}{$\begin{array}{l}\text { Stress-neutral } \\
\text { vs. Stress- } \\
\text { resilient }\end{array}$} & \multicolumn{2}{|c|}{$\begin{array}{l}\text { Stress-sensitive } \\
\text { vs. Stress- } \\
\text { resilient }\end{array}$} & \multicolumn{2}{|c|}{$\begin{array}{l}\text { Stress-neutral } \\
\text { vs. Stress- } \\
\text { sensitive }\end{array}$} \\
\hline & Estimate & SE & Estimate & SE & Estimate & SE \\
\hline Age & 0.06 & 0.04 & 0.01 & 0.03 & 0.06 & 0.03 \\
\hline Female & -0.39 & 0.21 & $0.54^{*}$ & 0.18 & $-0.93^{*}$ & 0.20 \\
\hline European American & 0.28 & 1.11 & -0.43 & 0.93 & 0.71 & 0.96 \\
\hline African American & -0.09 & 1.22 & -0.11 & 1.01 & 0.01 & 1.09 \\
\hline Latino & 1.99 & 1.23 & 0.07 & 1.03 & 1.92 & 1.07 \\
\hline Asian & 0.52 & 1.12 & -0.48 & 0.94 & 1.00 & 0.98 \\
\hline Multi-racial or Other & 0.69 & 1.14 & -0.50 & 0.98 & 1.187 & 1.00 \\
\hline $\begin{array}{l}\text { Positive history of } \\
\text { depression }\end{array}$ & $1.25^{*}$ & 0.22 & $0.95^{*}$ & 0.18 & 0.30 & 0.21 \\
\hline Family history & 0.32 & 0.20 & 0.15 & 0.17 & 0.17 & 0.19 \\
\hline Neuroticism & $0.23^{*}$ & 0.02 & $0.12^{*}$ & 0.02 & $0.11^{*}$ & 0.02 \\
\hline Early family environment & $0.05^{*}$ & 0.01 & $0.03^{*}$ & 0.01 & $0.03^{*}$ & 0.01 \\
\hline Internal medsicine & -0.26 & 0.26 & 0.22 & 0.22 & -0.48 & 0.26 \\
\hline Surgery & 0.52 & 0.37 & $0.76^{*}$ & 0.31 & -0.24 & 0.36 \\
\hline Pediatrics & -0.23 & 0.38 & 0.02 & 0.31 & -0.25 & 0.36 \\
\hline Psychiatry & 0.25 & 0.41 & 0.41 & 0.34 & -0.17 & 0.38 \\
\hline
\end{tabular}

$N=2240$.

Multinomial logistic regression are on the logit (log odds), and results should be interpreted with respect to the reference class which is the last class listed in each comparison.

$\mathrm{SE}$, standard error.

${ }^{*} P<0.05$.

neuroticism, adverse early family environment, female gender and surgical specialty predicted membership in the Stress-sensitive class, compared with the Stress-resilient Class. A personal history of depression, high neuroticism, and adverse early family environment also predicted membership in the Stress-neutral Class, compared with the Stressresilient Class. 


\section{Discussion}

Using the trajectory-based analysis, we demonstrate, for the first time, that there are distinct trajectories of depressive symptoms in response to a prolonged stressor, medical internship. Interns separate into three distinct classes of depressive symptoms during the stressful condition. Specifically, subjects in the largest trajectory class in our sample (Stress-resilient) report low depressive symptoms prior to the onset of stress and again throughout the year of high stress. Subjects in the second largest trajectory class (Stress-neutral) report mild depressive symptoms before the onset of stress and again throughout the year of stress. These groups are similar in that depressive symptoms do not fluctuate in response to stress, but differ in that the Stress-Neutral class is experiencing clinically mild depressive symptoms on an ongoing bases and the Stress-resilient class experiences no clinically relevant depressive symptoms. In contrast, subjects in the smallest trajectory class (Stress-sensitive) report low depressive symptoms before the onset of stress but high depressive symptoms throughout the year of stress. Over the course of internship year, $81 \%$ of the Stress-sensitive class had a PHQ9 depression score indicating a probable diagnosis of major depression during at least one follow-up assessment, compared with $53 \%$ and $4.2 \%$ of those in the Stress-neutral or Stress-resilient class, respectively.

In addition to identifying distinct classes of depressive symptoms under conditions of stress, we have also identified a number of important preinternship predictors of class membership. History of depression, high neuroticism, difficulty early family environment, female gender, and surgical specialty increased the likelihood of membership in the Stress-sensitive class, in comparison with the Stress-resilient class. Additionally, history of depression, high neuroticism, and adverse family environment increased likelihood of membership in the Stress-neutral class, characterized by mild depressive symptoms throughout the year, compared with the Stress-resilient class.

There are several important implications that follow from this study. First, our prospective study design and statistical approach has identified a subsample of individuals most vulnerable to depressive symptoms under conditions of stress. While prior studies have consistently demonstrated an overall association between stress and depression $(6-8,23,24)$, our findings suggest that the increase in depressive symptoms in response to stress is a precipitant to depressive symptoms in only a small subsample of individuals (Stress- sensitive class), rather than a common increase across the full population. Second, we identify a preinternship demographic and psychological profile that predicts membership in this high-risk subsample. Although the demographic and psychological profile has historically been shown to associate with depression (6-8), our prospective study design demonstrates that these factors predict a significant increase in depressive symptoms under conditions of stress. These findings have important clinical and health economic implications as it allows residency programs to focus limited prevention and intervention resources on the small subset of trainees most vulnerable to responding poorly to internship stress. If replicated in samples exposed to other forms of prolonged stressors (e.g., military members, patients' with chronic medical conditions), this finding will have broad utility for identification and resource allocation. For example, the incidence of depression for those with the profile found in the Stresssensitive class could be decreased by a targeted preventative intervention, which are most effective for those at greatest risk of the disease (25). It is important to note, however, that factors shown to predict class membership are only risk factors and do not determine an individual's course or trajectory for internship year. An alternative to a targeted prevention intervention is a universal screening within the first few months of internship with referral for clinically significant symptoms, as depressive symptoms that are high at 3 months of internship appear to remain elevated throughout internship year.

Beyond the clinical implication of these findings, growth mixture modeling captures important information about how individuals experience depressive symptoms under stress. For example, depression scores for the Stress-sensitive class from preinternship to internship year change dramatically, whereas for the other classes, they remained relatively stable across the year. If we employed standard regression analyses and looked at the overall population average, we would be missing this important variability and heterogeneity among groups. Growth mixture modeling identifies classes with more homogenous or more similar severity of depressive symptoms over time.

The identification of distinct trajectory classes with less fluctuation and greater stability in depressive symptoms can provide a more homogenous population for studies investigating the biological basis of depression. For instance, the Stresssensitive class of individuals may provide a platform to better understand the biologic effects of stress-induced depression. Similarly, investigating 


\section{Guille et al.}

biological markers in individuals demonstrating mild but persistent symptoms of depression over time, independent of environmental stressor (Stress-neutral), may provide an opportunity to gain insight into dysthymia, which likely has different biologic and genetic underpinnings compared with stress-induced depression. Investigating classes of individuals with depressive symptoms that are more homogenous will likely increase our ability to detect biological contributors to the disease (26).

While it is well established that women experience higher rates of depression compared with men $(1,8)$, there is conflicting evidence on whether the relationship between stress and depression differs between men and women (2730). In our study, we find that female gender increased the likelihood of membership in the Stress-sensitive class. This finding adds to an emerging pattern of results, with prospective studies finding evidence for gender difference in stress sensitivity and retrospective studies finding no evidence for gender difference, suggesting that recall bias may be playing an important role in the different results (30). The prospective study of stress and depression maybe an important direction for advancing our understanding of sex differences in depression (31).

There are several limitations to our study. First, our study was restricted to interns completing their first year of postgraduate medical training. Thus, it is unknown if similar classes of depressive symptom trajectories hold true for other populations experiencing a stressor. Second, only $59 \%$ of invited individuals chose to participate in the study. Previous work demonstrates minimal demographic differences in age and sex between those who chose to take part in the study and those who did not (13). However, other unmeasured factors may account for study participation (i.e., incentives or interest in the topic) or non-participation (i.e., lack of time) and could potentially be a source of study bias. Thus, our results should be extrapolated with caution. Third, while we included covariates in the study that have previously predicted increased depressive symptoms in medical interns (13), other potentially important predictors of depression in this population likely exist. Fourth, although multiple studies have demonstrated the validity, sensitivity, and specificity of the PHQ-9 inventory $(14,15)$, it is important to note that we assessed depression through a self-report inventory rather than a diagnostic interview. However, we chose this method as opposed to in person assessment given that medical trainees are less likely to honestly report depressive symptoms when assess- ments are not anonymous (32). Lastly, the employed modeling framework, although it has numerous strengths, is not without its limitations. One criticism of growth mixture models in general is that because standard chi-square difference tests cannot be used in this setting (33), formal tests of model fit to the data cannot be conducted (34). Therefore, growth mixture modeling cannot prove the existence of latent classes, but only describe the putative growth trajectory classes $(35,36,37)$. Additionally, although the determination of the trajectory classes are relatively robust to missing data, if data are not missing at random, the point estimates of the parameters may be biased, although it is unlikely that such bias would be substantial (38).

Our study provides evidence of distinct classes of depressive symptoms in response to stress and identifies a set of prestress factors that predict trajectory class membership. The trajectory-based analysis allows for the identification of a high-risk group in a heterogeneous population, a finding for which there is significant implications for clinical intervention and potentially provides an improved platform for identifying biological risk of stressrelated depression.

\section{Acknowledgement}

We would like to acknowledge and thank the interns taking part in this study. Without their participation this study would not be possible.

\section{Declaration of interests}

Constance Guille, Shaunna Clark, Ananda Amstadter and Srijan Sen have no competing or financial conflicts of interest to disclose. Research funds were provided by the National Institute of Health (NIH)/National Institute on Drug Abuse (NIDA) and Office of Research on Women's Health (ORWH) (P50 DA016511)-Specialized Centers of Research (SCOR) on Sex and Gender Factors Affecting Women's Health, National Institute of Mental Health (NIMH) (MH095109), an American Foundation for Suicide Prevention (AFSP) Young Investigator grant, National Institute of Alcohol Abuse and Alcoholism (NIAAA) (020179 and K01AA021266). NIH, NIDA, ORWH-SCOR, NIMH, AFSP, and NIAAA were not involved in the design and conduct of the study; collection, management, analysis, and interpretation of the data; and preparation, review, or approval of the manuscript. The content is solely the responsibility of the authors and does not necessarily represent the official views of NIH, NIDA, ORWH-SCOR, NIMH, AFSP, and NIAAA.

\section{References}

1. Kessler RC, Chiu WT, Demler O, Merikangas KR, Walters EE. Prevalence, severity, and comorbidity of 12-month DSM-IV disorders in the National Comorbidity Survey Replication. Arch Gen Psychiatry 2005;62:617-627. 
2. Dohrenwend BP. Sociocultural and social-psychological factors in the genesis of mental disorders. J Health Soc Behav 1975;16:362-365.

3. Brown GW, Harris TO, Hepworth C. Life events and endogenous depression. A puzzle reexamined. Arch Gen Psychiatry 1994;51:525-534.

4. Maciejewski PK, Mazure CM. Stressful life events and depression. Am J Psychiatry 2000;157:1344-1345.

5. Hammen C. Stress and depression. Annu Rev Clin Psychol 2005;1:293-319.

6. Boyce P, Parker G, Barnett B, Cooney M, Smith F. Personality as a vulnerability factor to depression. Br J Psychiatry 1991;159:106-114.

7. Akiskal HS, McKinney WT. Overview of recent research in depression. Integration of ten conceptual models into a comprehensive clinical frame. Arch Gen Psychiatry 1975; 32:285-305.

8. Kendler KS, Gardner CO, Prescott CA. Toward a comprehensive developmental model for major depression in men. Am J Psychiatry 2006;163:115-124.

9. Kendler KS, Gardner CO. Dependent stressful life events and prior depressive episodes in the prediction of major depression: the problem of causal inference in psychiatric epidemiology. Arch Gen Psychiatry 2010;67:1120-1127.

10. WANG J. Work stress as a risk factor for major depressive episode(s). Psychol Med 2005;35:865-871.

11. Thomas JL, Wilk JE, Riviere LA, McGurk D, Castro CA, Hoge CW. Prevalence of mental health problems and functional impairment among active component and National Guard soldiers 3 and 12 months following combat in Iraq. Arch Gen Psychiatry 2010;67:614-623.

12. Bellini LM, Baime M, Shea JA. Variation of mood and empathy during internship. JAMA 2002;287:3143-3146.

13. Sen S, Kranzler HK, Chan G et al. A prospective cohort study investigating factors associated with depression during medical internship. Arch Gen Psychiatry 2010;67: 557-565.

14. Spitzer RL, Kroenke K, Williams JB. Validation and utility of a self-report version of PRIME-MD: the PHQ primary care study. Primary Care Evaluation of Mental Disorders. Patient Health Questionnaire. JAMA 1999;282:17371744 .

15. Kroenke K, Sitzer RL, Williams JB. The PHQ-9: validity of a brief depression severity measure. J Gen Intern Med 2001;16:606-613.

16. Costa PT, McCrae RR. Stability and change in personality assessment: the revised NEO Personality Inventory in the year 2000. J Pers Assess 1997;68:86-94.

17. Taylor SE, Way BM, Welch WT, Hilmert CJ, Lehman BJ, EISENBERGER NI. Early family environment, current adversity, the serotonin transporter promoter polymorphism, and depressive symptomatology. Biol Psychiatry 2006;60: 671-676.

18. Clark DA, Steer RA, Beck AT, Ross L. Psychometric characteristics of revised Sociotropy and Autonomy Scales in college students. Behav Res Ther 1995;33:325-334.

19. Muthén LK, Muthén BO. MPlus User's Guide. 6th edn. Los Angeles: Muthen \& Muthen, 1998-2010.

20. McLachlan G, Peel D. Finite Mixture Models. 2000, New York: Wiley... A new look at the statistical model identification. Automatic Control, IEEE Transactions 1974; 19:716-723... Estimating the Dimension of a Model. Annals of Statistics 1978;6:461-464.
21. Ormel J, Oldehinkel AJ, Brilman EI. The interplay and etiological continuity of neuroticism, difficulties, and life events in the etiology of major and subsyn- dromal, first and recurrent depressive episodes in later life. Am J Psychiatry $2001 ; \mathbf{1 5 8}: 885-891$.

22. HARRIS T. Recent developments in understanding the psychosocial aspects of depression. Br Med Bull 2001;57: $17-32$.

23. Muñoz R, Cuijpers P, Smit F, Barrera A, Leykin Y. Prevention of major depression. Annu Rev Clin Psychol 2010;6:181-212.

24. Kerner B, Muthén BO. Growth-mixture modeling in families of the Framingham Heart Study. BMC Proc 2009;3: S114.

25. Kessler RC, McGonagle KA, Swartz M, Blazer DG, Nelson CB. Sex and depression in the National Comorbidity Survey I: lifetime prevalence, chronicity and recurrence. J Affect Disord 1993;29:85-96.

26. Kendler KS, Gardner CO, Prescott CA. Toward a Comprehensive Model for Major Depression in Women. Am J Psychiatry 2002;159:1133-1145.

27. Hyde JS, Mezulis AH, Abramson LY. The ABCs of depression: integrating affective, biological, and cognitive models to explain the emergence of the gender difference in depression. Psychol Rev 2008;115:291-313.

28. Oldehinkel AJ, Bouma EMC. Sensitivity to the depressogenic effect of stress and HPA-axis reactivity in adolescence: a review of gender differences. Neurosci Biobehav Rev 2010;35:1757-1770.

29. Melchior M, Chastang JF, Head J et al. Socioeconomic position predicts long-term depression trajectory: a 13-year follow-up of the GAZEL cohort study. Mol Psychiatry 2011;1:1-10.

30. Levine RE, Breitkopf CR, Sierles FS, Camp G. Complications associated with surveying medical student depression-the importance of anonymity. Acad Psychiatry 2003; 27:12-18.

31. Mclachlan G, Peel D. Finite Mixture Models. New York: Wiley, 2000.

32. Vermunt JK, Magidson J. Latent class analysis. In: LewisBeck M, Bryman A, Liao T, eds. The sage encyclopedia of social science research methods. Newbury Park: Sage, 2003:550-554.

33. Vermunt JK, Magidson J. Latent class cluster analysis. In: HagenaArs J, McCutcheon A, eds. Applied latent class analysis. Cambridge, UK: Cambridge Univeristy Press, 2002:89-106.

34. Borboom D, Mellenbergh GJ, van Heerden J. The theoretical status of latent variables. Psychol Rev 2003;110:203219.

35. Steinley D, Brusco MJ. Evaluating mixture modeling for clustering: recommendations and cautions. Psychol Methods 2011;16:63-79.

36. Borboom D, Mellenbergh GJ, van Heerden J. The theoretical status of latent variables. Psychol Review 2003;110: 203-219.

37. Steinley D, Brusco MJ. Evaluating mixture modeling for clustering: Recommedations and cautions. Psychol Methods 2011;16: 63-79.

38. Muthén B, Kaplan D, Hollis M. On structural equation modeling with data that are not missing completely at random. Psychometrika 1987;42:431-462. 